\title{
Directions and Magnitudes of Misregistration of CT Attenuation-Corrected Myocardial Perfusion Studies: Incidence, Impact on Image Quality, and Guidance for Reregistration
}

\author{
John A. Kennedy ${ }^{1}$, Ora Israel ${ }^{1,2}$, and Alex Frenkel ${ }^{1}$
}

${ }^{I}$ Department of Nuclear Medicine, Rambam Health Care Campus, Haifa, Israel; and ${ }^{2}$ B. and R. Rappaport School of Medicine, Technion, Israel Institute of Technology, Haifa, Israel

CT-based attenuation-corrected (AC) myocardial perfusion imaging (MPI) studies may show significant artifacts caused by misregistration between SPECT and CT data. The present study aimed at identifying the directions and magnitudes of misregistration with greatest impact on AC myocardial perfusion image quality. Methods: The incidence, magnitude, and direction of misregistration were assessed in 248 consecutive stress-rest MPI studies in 124 patients. In addition, cardiac SPECT/CT registration was artificially modified in 40 studies, shifting CT data by $\pm 1, \pm 2$, and \pm 3 pixels along the cephalad/caudal, dorsal/ventral, and left/right axes. Percentage of change in 5-wall AC-MPI polar map scoring was calculated for each region, and the direction of the shift along each axis was analyzed statistically (Student $t$ test, $P<0.05$ ) and compared to determine the region most significantly affected by each shift (Newman-Keuls test, $P<0.05)$. Changes in the normal and abnormal summed stress score (SSS) due to artificial misregistration were assessed ( $\kappa$-statistics, McNemar differences). Results: SPECT/CT misregistration of more than 1 pixel was found in $73 \%(181 / 248)$ of studies and more than 2 pixels in $23 \%$ of studies (57/248). A 3pixel ventral shift most significantly affected polar map scoring $(-15.4 \% \pm 6.1 \%$ change in lateral wall; $-7.5 \% \pm 5.5 \%$ change in inferior wall). A 3-pixel dorsal shift resulted in a $-9.5 \% \pm 5.3 \%$ apical and $-8.8 \% \pm 5.8 \%$ septal change. Polar map scoring was least affected by the cephalad/caudal shift $(<5 \%$ average change in all regions except for the anterior wall; $-9.9 \% \pm$ $7.4 \%$ change for 3-pixel caudal shift). The most significant changes occurred in the lateral and anterior walls when the myocardium on SPECT overlapped lung tissue on CT, encountered in $16 \%$ of studies (40/248). Clinically significant changes (in SSS) occurred for the 3-pixel caudal, dorsal, and right shifts. Conclusion: A misregistration of significant magnitude occurred in $23 \%$ of studies and in the direction of the most severe artifacts in $16 \%$ of studies. Severe misregistration along the dorsal/ventral axis most significantly affected AC-MPI. Quality control of

Received Jan. 14, 2009; revision accepted Jun. 9, 2009.

For correspondence or reprints contact: John A. Kennedy, Department of Nuclear Medicine, Rambam Health Care Campus, P.O. Box 9602, Haifa, Israel 31096.

E-mail: j_kennedy@rambam.health.gov.il

COPYRIGHT $\odot 2009$ by the Society of Nuclear Medicine, Inc.
SPECT and CT registration and manual realignment should be performed routinely, with the highest priority in AC studies showing an overlay of the myocardium on SPECT with lung tissue on the CT component of AC-MPI.

Key Words: SPECT/CT; attenuation correction; misregistration; myocardial perfusion imaging

J Nucl Med 2009; 50:1471-1478

DOI: 10.2967/jnumed.109.062141

C linically significant artifacts on CT-based attenuationcorrected (AC) myocardial perfusion imaging (MPI) studies may be caused by misregistration of 1 pixel or more between the SPECT and CT data (1). The present study was performed to identify the directions and magnitudes of misregistration that have the greatest effect on $\mathrm{AC}$ myocardial perfusion image quality.

Hybrid SPECT/CT scanners provide an efficient method of obtaining CT-based AC-MPI $(2,3)$. Frequent artifacts seen on MPI studies include an apparent reduction in radiotracer uptake due to breast attenuation in women or to diaphragmatic attenuation, more often found in men (4). These findings are reduced or removed in AC-MPI, with subsequent improved diagnostic accuracy, in particular in patients with multivessel disease and reduced inter- and intraobserver variability $(5,6)$. Although clinical acceptance of AC is growing, AC can at times be related to patient motion and induce artifacts such as apical thinning. Arguments against using AC with CT because of increased capital costs and higher patient radiation exposure and the fact that misregistration between the transmission (CT) and emission (SPECT) scans can induce artifacts have caused concern $(1,7-11)$.

Goetze et al. (12) have demonstrated the value of software tools that enable operator realignment of misregistered 
SPECT and CT for avoiding artifacts. The advent of routinely available software for reregistration raises, however, the question of which directions of misalignment between SPECT and CT most severely affect AC myocardial perfusion image quality. Changes in relative regional myocardial tracer uptake as a function of misalignment of AC performed with a radioactive line source $(13,14)$ or coregistered from separately performed SPECT and CT (15) have been previously reported. There is, however, only a paucity of literature regarding frequency of misregistration, the potential relationship between its location and effect on image quality, and guidelines for reregistration of transmission-emission hybrid cardiac SPECT/CT. Consequently, the aim of this study was to define the incidence, direction, and severity of misregistration with the highest impact on image accuracy in hybrid SPECT/CT-acquired AC-MPI.

\section{MATERIALS AND METHODS}

\section{Patient Population}

This study includes a retrospective analysis of MPI studies performed on 144 patients. Approval for this study was received from the Helsinki committee of our institution. Two patient groups were evaluated in the current study. The first group included 248 consecutive stress-rest MPI studies performed on 124 patients (60 men, 64 women; mean age $\pm \mathrm{SD}, 62 \pm 12$ y) to assess the incidence, magnitude, and direction of misregistration. In a second study group, cardiac SPECT/CT registration was artificially modified to determine the effect of misregistration on AC myocardial perfusion image quality. This second group consisted of 20 randomly chosen patients (14 men, 6 women; mean age \pm SD, $59 \pm 13 \mathrm{y}$; mean body mass index $\pm \mathrm{SD}, 29.3 \pm 4.1 \mathrm{~kg} / \mathrm{m}^{2}$ ). Patients were referred for imaging to evaluate the presence of ischemic heart disease because of the following clinical indications: atypical chest pain $(n=8)$, typical chest pain $(n=4)$, myocardial infarction $(n=3)$, hypertension $(n=2)$, diabetes mellitus $(n=1)$, and hypertension with diabetes mellitus $(n=2)$. The likelihood of coronary artery disease (CAD) was high for 9 patients ( 2 had previously diagnosed CAD), medium for 5 patients, and low for 6 patients, as determined by an expert in cardiac rehabilitation and nuclear cardiology with $11 \mathrm{y}$ of clinical experience. Fifteen MPI studies were reported as showing normal findings and 5 as showing abnormal findings, including a scar involving the inferior wall in the first patient, inferoapical ischemia in the second patient, and ischemia and scarring in the anterior, anteroapical, and apical regions of the third, fourth, and fifth patients, respectively.

\section{Imaging Protocol}

Each patient underwent a single-day rest-stress ${ }^{99 \mathrm{~m}} \mathrm{Tc}$-sestamibi protocol with an injected dose of $370 \mathrm{MBq}$ for rest and 1,110 $\mathrm{MBq}$ for stress. The time difference between rest and stress studies was $2.3 \pm 0.6 \mathrm{~h}$. AC-MPI studies were performed on a VG or Infinia Hawkeye-1 SPECT/CT camera (referred to, respectively, as cameras 1 and 2) (GE Healthcare). The acquisition parameters were identical, as were the AC hardware and software used for these 2 imaging devices. SPECT was performed first, followed immediately by a low-current $\mathrm{CT}$ scan, scaled and filtered to provide an attenuation map. Patients were instructed to breathe normally during both the SPECT and the CT scans. AC-MPI studies are the clinical routine in the department. SPECT was performed with the 2 detectors in L-mode position, with 60 frames of $3^{\circ}$ (30 steps over a $180^{\circ} \mathrm{scan}$ ), for $25 \mathrm{~s}$ each for rest and $20 \mathrm{~s}$ each for stress. A $64 \times 64$ projection matrix of $6.8-\mathrm{mm}^{2}$ pixels was used. Stress data were electrocardiogram-gated into 8 bins. Singleslice CT data were acquired immediately after SPECT, using a tube current of $2.5 \mathrm{~mA}$ and a voltage of $140 \mathrm{kVp}$ for an axial field of view covering the myocardium in 13-17 slices of $10-\mathrm{mm}$ thickness over $5 \mathrm{~min}$. No breath holding or contrast agents were used.

Images were reconstructed using a maximum-likelihood expectation maximization $(16,17)$ algorithm ( 2 iterations, 10 subsets; transaxial pixel size, $3.4 \mathrm{~mm}$ ) and processed on a dedicated workstation (Xeleris; GE Healthcare). Processing included filtering (Butterworth; cutoff frequency, 0.4; order, 10) and resampling the data along the short, vertical long, and horizontal long axes for display.

Before iterative reconstruction with attenuation correction, registration of the myocardial wall between SPECT and CT in the transverse, coronal, and sagittal image planes was checked using the manufacturer's software (ACQC tool; GE Healthcare). If the isocontours of SPECT did not coincide with CT, the software enabled the operator to manually shift the CT image with respect to the SPECT image within the 3 planes for realignment to within less than 1 pixel, visually. The registration was recorded in a screen capture, which also indicated the magnitude of the reregistration applied (in millimeters) in the left/right ( $x$-axis), dorsal/ventral ( $y$-axis), and cephalad/caudal (z-axis) directions. These images were further available to the reporting physician for review, for the purpose of quality control (QC), during the reading session (Fig. 1).

\section{Data Acquisition and Analysis}

Of the 248 consecutive stress-rest MPI studies from the first patient study group, 114 were performed on camera 1 and 134 on camera 2. The initial registration data for the 124 patients were retained for further analysis of the frequency of misregistration. The magnitude of misregistration was assessed according to the number of pixels or axes by a single reader, a medical physicist with $2 \mathrm{y}$ of experience in reading fused cardiac SPECT and CT images. The frequency of misregistration of 1 pixel or more was assessed with respect to magnitude and direction in all 248 studies. Because stress-rest pairs of AC-MPI studies undergo comparative analysis for diagnosis, the incidence of incongruence in misregistration of 1 pixel or more between stress and rest studies performed on the same day was determined.

To study the effect of misregistration on the myocardial radiotracer uptake, registration of cardiac SPECT/CT images was modified in the stress and rest studies of 20 randomly chosen patients (16 studies performed on camera 1 and 24 on camera 2). The motion of the heart during SPECT acquisition was less than 1 pixel, as determined by the examination of the projection data; therefore, motion-correction software was not applied to these 20 patients. The SPECT and CT data were modified only in the laboratory, retrospectively, after the studies had been read and the clinical report had been completed, and were not used for clinical purposes. In these 40 studies, the registered CT data were artificially shifted by $\pm 1, \pm 2$, and \pm 3 pixels along the $x$-, $y$-, and $z$-axes (Fig. 2).

A 5-wall polar perfusion map score was calculated for each study using the Quantitative Perfusion SPECT/Quantitative Gated 


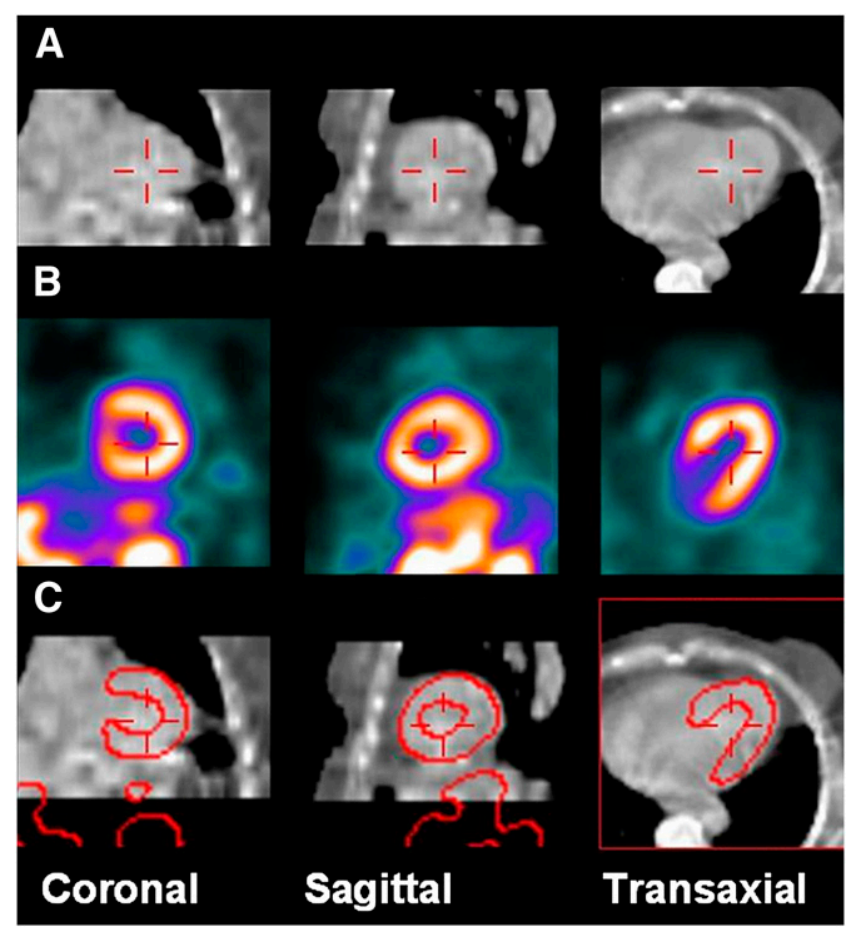

FIGURE 1. QC images for SPECT/CT registration in ACMPI. (A) CT: Hounsfield units obtained from CT are converted to appropriate attenuation coefficient values. (B) Non-AC-MPI. (C) Fused SPECT/CT: MPI contours are shown in red. Software-enabled control and correction of emission-transmission registration before $\mathrm{AC}$ image reconstruction.

SPECT (QPS/QGS) software (Cedars-Sinai Medical Center). This score represents the average radiotracer uptake in a segment, expressed as percentage of maximum voxel value in the myocardium. The percentage change in the polar map score was calculated for each of the 5 segments-anterior, apical, inferior, septal, and lateral walls-in all studies. The relative effects of misregis- tration on polar map scoring were categorized (differences of $<5 \%$, differences of $\geq 5 \%$ and $<15 \%$, or differences of $\geq 15 \%$ ) for the affected segments.

The changing characterization of a stress study from normal to abnormal or vice versa was evaluated using a 20-segment QPS/ QGS summed stress score (SSS) before and after misregistration for all axes and magnitudes. An SSS of less than 4 was considered to represent a normal study (18).

\section{Statistical Analysis}

For each shift, the percentage changes in the 5 segments were statistically analyzed using a Newman-Keuls multicomparison test performed after a significant ANOVA test $(P<0.05)$ to determine which region differed significantly from the others. Along each axis, for each segment, the results from the samemagnitude pixel shifts of opposite directions were compared by means of a Student $t$ test $(P<0.05)$ to determine whether the direction of the misregistration along the axis caused significantly different results. The statistical significance of the change in SSS from normal to abnormal or vice versa was assessed using к-statistics and McNemar differences.

\section{RESULTS}

SPECT/CT misregistration of greater than 1 pixel was found in 181 of the 248 studies, for a frequency of $73 \%$ (Table 1). Misregistrations of more than 1 pixel along more than 1 axis, or misregistrations of more than 3 pixels, were found in 28\% (69/248) of the studies. Differences in misregistration of more than 1 pixel along at least 1 axis between the stress and the rest study of the same patient were found in 36\% (45/124) of patients. Of the 23\% (57/248) of studies that had a misregistration of more than 2 pixels, in $16 \%(40 / 248)$ of these studies this misregistration occurred in the directions further demonstrated as causing the most severe changes.

The most significant effect on polar map scoring was a $-15.4 \% \pm 6.1 \%$ change in the lateral wall caused by a 3-pixel ventral shift, statistically significantly different from
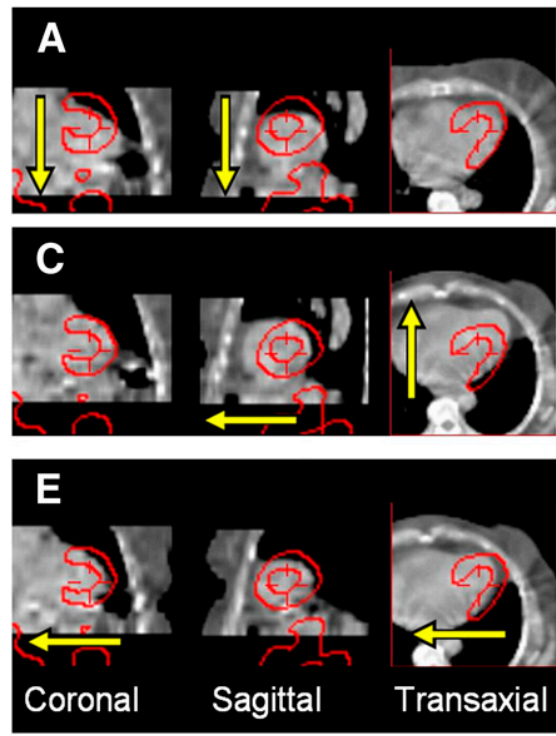
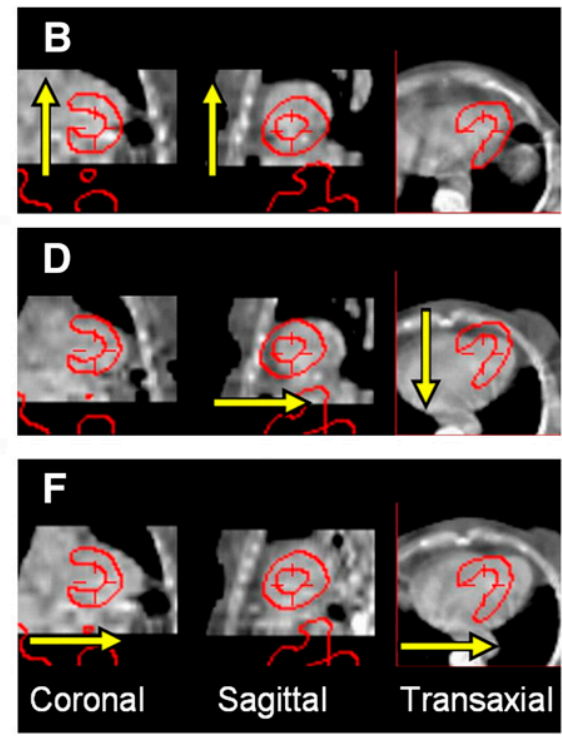

FIGURE 2. Artificial CT shift along 3 axes and 6 directions by 3 pixels with respect to SPECT (red contour). Directions were caudal, $-z$-axis $(A)$, cephalad, +z-axis (B), ventral, $-y$-axis (C), dorsal, $+y$-axis (D), right, $-x$-axis $(E)$, and left, $+x$-axis $(F)$. 
TABLE 1. Number of Studies with Given Direction and Magnitude of Misregistration

Direction of misregistration

On any axis

Most severe

direction

on any axis ${ }^{\star}$

$\geq 2$ axes

Most severe

direction $^{*}$

$\geq 2$ axes

*In different experiment, caudal, ventral, or right artificial shift of CT was found to induce most severe changes in polar map scoring.

Number in parentheses is percentage of 248 consecutive clinical studies.

the results for the other 4 segments (Newman-Keuls test, $P=0.0001$ ). The 3-pixel ventral shift also resulted in a $-7.5 \% \pm 5.5 \%$ change in the inferior wall. In the opposite direction, the 3-pixel dorsal shift resulted in a $-9.5 \% \pm$ $5.3 \%$ change in the apex and a $-8.8 \% \pm 5.8 \%$ change in the septum (Fig. 3).

For each axis, the largest significantly different change (Newman-Keuls test, $P=0.0001$ ) was caused by a 3-pixel shift of the CT scan from a registered position with respect to the SPECT scan: in the anterior wall by a caudal shift $(-9.9 \% \pm 7.4 \%)$, in the lateral wall by a ventral shift, and in the lateral wall by a right shift $(-8.7 \% \pm 5.3 \%)$. The 3-pixel caudal shift caused the anterior wall isocontours on SPECT to overlap lung tissue on CT, and the 3-pixel ventral and right shifts caused the lateral wall isocontours on SPECT to overlap lung tissue on CT (Fig. 2).

The relative effects of misregistration on polar map scoring according to intensity and segments demonstrate the presence of changes of less than 5\% in 17 segments, changes of at least $5 \%$ but less than $15 \%$ in 12 segments, and changes of $15 \%$ or more in 1 segment (Table 2). Of the 13 changes that were $5 \%$ or more, 2 were caused by the cephalad/caudal shifts, 3 by the left/right shifts, and 8 by the dorsal/ventral shifts (Table 2).

The change in characterization of the stress studies from normal to abnormal or vice versa, measured by SSS, was clinically significant for artificial misregistration of 3 pixels in the caudal $(\kappa=0.043$, McNemar $P=0.046$ ), dorsal $(\kappa=-0.075$, McNemar $P=0.027)$, and right shifts $(\kappa=$ 0.140, McNemar $P=0.013$ ) (Table 3).

The direction of artificial misregistration along an axis caused significantly different results in 10 of the 15 cases ( 3 axes and 5 segments). CT misregistration of 3 pixels did not give statistically significant differences in polar map scores between the positive and negative $z$-axis directions for the septum $(0.57 \% \pm 7.02 \%$ vs. $-1.24 \% \pm 5.90 \%, P=0.111)$
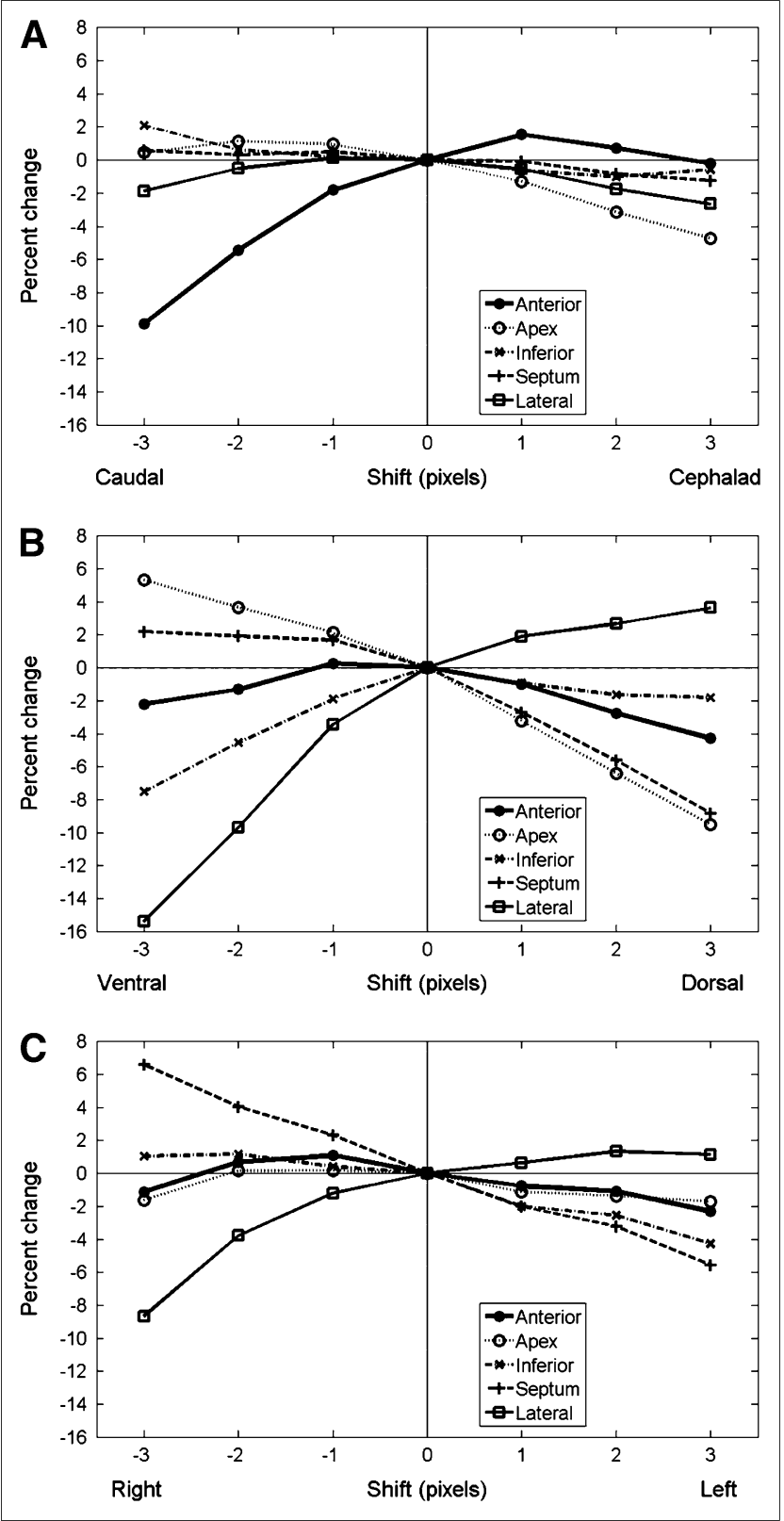

FIGURE 3. Percentage change in polar map segment score as function of misregistration of CT with respect to SPECT. For each axis, most severe changes are caused by 3-pixel shift as follows: anterior wall by a caudal shift $(A)$, lateral wall by ventral shift $(B)$, and lateral wall by right shift (C). All severe changes can be visually identified as misregistration, with myocardium on SPECT overlapping lung tissue on CT.

and lateral wall $(-1.88 \% \pm 6.41 \%$ vs. $-2.64 \% \pm 4.94 \%$, $P=0.278$ ). For the anterior wall, the direction along the $y$ and $x$-axes did not provide significantly different results $(-4.28 \% \pm 5.06 \%$ vs. $-2.20 \% \pm 6.29 \%, P=0.056$, and $-2.31 \% \pm 4.84 \%$ vs. $-1.11 \% \pm 5.73 \%, P=0.159)$, nor did the \pm 3 -pixel $x$-axis shifts for the apex $(-1.70 \% \pm$ $4.40 \%$ vs. $-1.63 \% \pm 6.37 \%, P=0.479$ ) (Table 4$)$. 
TABLE 2. Relative Effect of CT Misregistration on Change in AC-MPI Polar Map Score

\begin{tabular}{|c|c|c|c|c|c|c|}
\hline \multirow[b]{2}{*}{ Direction of misregistration } & \multirow{2}{*}{$\begin{array}{c}\text { Magnitude of } \\
\text { misregistration (pixels) }\end{array}$} & \multicolumn{5}{|c|}{ Region } \\
\hline & & Anterior & Apex & Inferior & Septum & Lateral \\
\hline \multirow[t]{3}{*}{ Cephalad (+z-axis) } & 3 & - & - & - & - & - \\
\hline & 2 & + & - & - & - & - \\
\hline & 1 & + & - & - & - & - \\
\hline \multirow[t]{3}{*}{ Caudal (-z-axis) } & 1 & - & + & + & + & + \\
\hline & 2 & -- & + & + & + & - \\
\hline & 3 & -- & + & + & + & - \\
\hline \multirow[t]{3}{*}{ Dorsal (+y-axis) } & 3 & - & -- & - & -- & + \\
\hline & 2 & - & -- & - & -- & + \\
\hline & 1 & - & - & - & - & + \\
\hline \multirow[t]{3}{*}{ Ventral (-y-axis) } & 1 & - & + & - & + & - \\
\hline & 2 & - & + & - & + & -- \\
\hline & 3 & - & ++ & -- & + & --- \\
\hline \multirow[t]{3}{*}{ Left $(+x$-axis) } & 3 & - & - & - & -- & + \\
\hline & 2 & - & - & - & - & + \\
\hline & 1 & - & - & - & - & + \\
\hline \multirow[t]{3}{*}{ Right (- $x$-axis) } & 1 & + & + & + & + & - \\
\hline & 2 & + & + & + & + & - \\
\hline & 3 & - & - & + & ++ & -- \\
\hline \multicolumn{7}{|c|}{$\begin{array}{l}\text { Difference with respect to registered SPECT/CT: }+++=\text { positive change of } \geq 15 \% ;---=\text { negative change of } \leq-15 \% ;++= \\
\text { positive change of }<15 \% \text { and } \geq 5 \% ;--=\text { negative change of }>-15 \% \text { and } \leq-5 \% ;+=\text { positive change of }<5 \% \text { and }>0 \% \text {; }-= \\
\text { negative change of }>-5 \% \text { and }<0 \% \text {. }\end{array}$} \\
\hline
\end{tabular}

\section{DISCUSSION}

Misregistration of 1 pixel or more between cardiac SPECT and CT scans can cause artifacts in AC-MPI, with further impact on image interpretation (10). Artifactual defects due to SPECT/CT misregistration in AC-MPI have been reported for the septum (I) and for the apical and anterior walls $(1,9)$ but have not been related uniquely to misregistration and can be caused by additional factors. It is, therefore, important to check the degree of registration as a routine QC step. In the present study, misregistration of 1 pixel or more was found with an incidence of 73\% (181/ 248 ), not significantly different from the previously reported 64\% (128/199) $(P=0.062)(12)$, and misregistration of more than 2 pixels was found with an incidence of $23 \%$ (57/248), higher than the 7\% (15/199) reported by the same authors $(P<0.0001)(12)$. Although the reason for these different results is not clear, they may be due to registration bias in individual imaging devices, differing degrees of patient motion, or table flexion. The current study demonstrates that significant $(>1$ pixel) misregistration can occur in more than 1 direction. These findings emphasize the need to define methods of reducing such contributing factors, to develop software methods for registration QC, and to prioritize manual correction.

The method of registration modification used in the present study is the best practice recommended to date. The study addresses the application of the AC-QC tool for $\mathrm{QC}$ of SPECT/CT registration and realignment in clinical routine. Phantom studies of misregistration have been previously described (15). However, studies using a phantom would not show the variability found in clinical studies because phantoms do not exhibit natural patient motion such as breathing and do not have the wide range of tissue thicknesses and densities found in a patient population showing the known variability in human anatomy. Therefore, in the present review random patient studies were selected to assess the effects of misregistration on a typical population being clinically evaluated. Motion QC is performed as a rule by examining projection data, and if needed, motion is corrected using semiautomatic software before registration QC and therefore does not contribute to misregistration between the emission and the transmission maps. However, patient motion during an emission scan is a contributing factor to poor image quality; therefore, a patient population rather than a phantom was chosen for this study.

The present study indicates that for a given magnitude, the direction of misregistration can cause significantly different results (Tables 2 and 4). A ventral or right shift leads to a decrease in uptake in the lateral wall, whereas a caudal shift leads to a decrease in uptake in the anterior wall. These severe changes coincide with and can be visually identified as misregistration, with the myocardium on SPECT overlapping lung tissue on CT. Similarly, the dorsal shift causes the apex and septum in SPECT to overlap less-dense fatty tissue in the anterior mediastinum in CT. The assigned attenuation coefficient values for lung and fat are lower than those for the myocardium and the cause of the lateral, septal, 


\begin{tabular}{|c|c|c|c|c|c|}
\hline \multirow[b]{2}{*}{ Misregistration direction } & \multirow[b]{2}{*}{$\begin{array}{l}\text { Misregistration } \\
\text { magnitude (pixels) }\end{array}$} & \multicolumn{2}{|c|}{$\begin{array}{l}\text { No. of patients changing } \\
\text { category }^{\star}\end{array}$} & \multirow[b]{2}{*}{ к-value } & \multirow[b]{2}{*}{ McNemar test } \\
\hline & & $\begin{array}{c}\text { Abnormal to } \\
\text { normal }\end{array}$ & $\begin{array}{l}\text { Normal to } \\
\text { abnormal }\end{array}$ & & \\
\hline \multirow[t]{3}{*}{ Cephalad (+z-axis) } & 3 & 2 & 3 & 0.440 & NS \\
\hline & 2 & 4 & 1 & 0.510 & NS \\
\hline & 1 & 4 & 3 & 0.300 & NS \\
\hline \multirow[t]{3}{*}{ Caudal (-z-axis) } & 1 & 3 & 0 & 0.706 & NS \\
\hline & 2 & 3 & 3 & 0.394 & NS \\
\hline & 3 & 2 & 7 & 0.043 & $P<0.05$ \\
\hline \multirow[t]{3}{*}{ Dorsal (+y-axis) } & 3 & 2 & 8 & -0.075 & $P<0.05$ \\
\hline & 2 & 1 & 7 & 0.400 & $P<0.05$ \\
\hline & 1 & 3 & 4 & 0.286 & NS \\
\hline \multirow[t]{3}{*}{ Ventral (-y-axis) } & 1 & 4 & 1 & 0.510 & NS \\
\hline & 2 & 4 & 2 & 0.406 & NS \\
\hline & 3 & 2 & 3 & 0.490 & NS \\
\hline \multirow[t]{3}{*}{ Left ( $+x$-axis) } & 3 & 3 & 4 & 0.286 & NS \\
\hline & 2 & 2 & 2 & 0.596 & NS \\
\hline & 1 & 3 & 1 & 0.604 & NS \\
\hline \multirow[t]{3}{*}{ Right $(-x$-axis $)$} & 1 & 3 & 2 & 0.500 & NS \\
\hline & 2 & 2 & 4 & 0.381 & NS \\
\hline & 3 & 1 & 7 & 0.140 & $P<0.05$ \\
\hline
\end{tabular}

${ }^{*}$ Registered case: 9 normal (SSS $\left.<4\right), 11$ abnormal (SSS $\left.\geq 4\right)$.

NS $=$ no significant difference $(P>0.05)$.

and apical wall artifacts in respective AC-MPI. Changes in tracer uptake in the lateral wall and septum due to overlap with lung tissue have been previously reported using radionuclide transmission sources $(13,14)$, but decreased uptake in the anterior wall for a caudal shift as found in the present study was not consistently demonstrated, potentially because of differences in the previously used phantom and the assessed patient population.

Misregistration along the dorsal/ventral axes affected accurate scoring in 4 of the 5 segments (Table 2), causing changes of $5 \%$ or more in the apical, inferior, septal, and lateral walls, whereas only the anterior wall was affected by a less than $5 \%$ change. In contrast, the caudal shift was the only $z$-axis shift causing a change of at least $5 \%$ but less than $15 \%$ and reduced the score in the anterior wall. The 3pixel left/right misregistration caused changes of at least $5 \%$ but less than $15 \%$ in the septum in either direction and in the lateral wall for the $-x$-axis (right) direction. In contrast to the results obtained by measuring the percentage change in polar map score, the 3-pixel dorsal shift, rather than ventral shift, gave the most significant change along that axis according to the analysis of the change in SSSmeasured normal or abnormal clinical grouping. The 3pixel caudal and right shifts gave the most significant change in normal or abnormal SSS grouping, consistent with the results obtained by measuring the percentage change in polar map score (Table 3).

CT-based AC used in a previous phantom study (15) did not identify the dorsal/ventral registration as having the most important effect with respect to AC myocardial perfusion image quality. The finding of this previous study may be explained by the less appropriate structure of the phantom (neither lung tissue nor mediastinal fat with density less than the myocardium was modeled immediately dorsal to the lateral wall) and by the fact that separate SPECT and CT studies were coregistered using fiducial markers.

The present study addresses the misregistration of the radiotracer activity and the anatomic structures used for the

TABLE 4. Directionality: Misregistrations with Significant Difference Between Positive and Negative 3-Pixel Shifts (by Student $t$ test)

\begin{tabular}{lccccc}
\multicolumn{1}{c}{$\begin{array}{c}\text { Misregistration } \\
\text { direction }\end{array}$} & \multicolumn{4}{c}{ Region } \\
\cline { 2 - 6 } Cephalad/caudal (z-axis) & Anterior & Apex & Inferior & Septum & Lateral \\
Dorsal/ventral $(y$-axis) & NS & $P<0.001$ & $P<0.025$ & NS & $P<0.001$ \\
Left/right $(x$-axis) & NS & NS & $P<0.001$ & $P<0.001$ & $P<0.004$ \\
NS $=$ no significant difference between positive and negative shifts $(P>0.05)$. &
\end{tabular}


attenuation map. High subdiaphragmatic tracer activity may be encountered often and is clinically significant but relates to radiotracer activity in 2 different organs. For the current analysis, the radiotracer activity in different organs was not misregistered relative to each other.

The axial mismatches between the attenuation and the emission maps caused by breathing have been shown to cause artifacts, especially in the anterior wall, in both PET and SPECT MPI $(19,20)$. Reregistration of such misalignment in SPECT/CT leads to superior image quality if based on internal rather than external landmarks, whether performed with a free-breathing CT protocol or during breath holding after a normal exhalation (20). Analysis of misregistration in cardiac PET/CT has also indicated that artifactual defects due to misregistration can be substantially reduced using average CT attenuation data during a normal breathing cycle (21) and manually adjusting the data for visual coregistration with PET when necessary (21-23).

Misregistration artifacts in AC-MPI performed with PET/ CT are more frequent and severe for helical CT-based than for radionuclide-based transmission maps, in part because of the slower acquisition time of the latter, which averages the attenuation values during a breathing cycle (21). The relatively longer duration of the CT acquisition of the SPECT/CT systems used in the present study likely ameliorates the negative effects of breathing-related misregistration.

With the increasing use of hybrid SPECT/CT systems in nuclear cardiology, knowledge and quantification of the relative effect of SPECT and CT misregistration on AC myocardial perfusion image quality, as enabled by the present data obtained from a large number of studies (40 studies in 20 patients), becomes increasingly clinically significant. Table 2 provides a look-up guide to determine the relative effects of misregistration on the AC-MPI radiotracer uptake in 5 myocardial segments for the purpose of AC-QC and reregistration in the clinical setting. Compared with the other 2 axes, dorsal/ventral misregistration is responsible for a greater degradation of AC myocardial perfusion image quality as reflected by the larger number of polar map scoring changes that were $5 \%$ or more. Reregistration should, therefore, be performed to ensure that the myocardial wall on SPECT does not overlap lung tissue on $\mathrm{CT}$, especially along the dorsal/ventral axis. When correction for misregistration cannot be performed satisfactorily, findings should be based on non-AC reports only.

The scope of this study-limited to assessing the effects of misregistration on AC myocardial perfusion image quality - did not aim at performing a comprehensive analysis of diagnostic significance. The results present the averaged effect and show that misregistration can cause a diagnostic problem in a general patient population. The effect of misregistration due to rotation, and the results of its reregistration, have not been addressed in the present article and are currently considered the subject for a future project.

\section{CONCLUSION}

A severe misregistration along the dorsal/ventral axis was found to most significantly affect AC myocardial perfusion image quality. Clinically significant SPECT/CT misregistration of severe magnitude ( $>2$ pixels) occurred in $23 \%$ of studies and specifically in the direction leading to the most severe artifacts in $16 \%$ of studies. Results of the present study provide guidance before the performance of manual realignment using registration software tools, demonstrating that priority should be given to cases showing overlay of the myocardium on SPECT with lung tissue on the CT component of AC-MPI studies.

\section{ACKNOWLEDGMENTS}

We acknowledge the clinical contribution of Dr. Shmuel Rispler in defining the likelihood of CAD in our patient population and of Kinga Przewloka in collecting and entering data.

\section{REFERENCES}

1. Tonge CM, Manoharan M, Lawson RS, Shields RA, Prescott MC. Attenuation correction of myocardial SPECT studies using low resolution computed tomography images. Nucl Med Commun. 2005;26:231-237.

2. Masood Y, Liu Y, DePuey G, et al. Clinical validation of SPECT attenuation correction using $\mathrm{X}$-ray computed tomography-derived attenuation maps: multicenter clinical trial with angiographic correlation. J Nucl Cardiol. 2005;12: 676-686.

3. Heller GV, Links J, Bateman TM, et al. American Society of Nuclear Cardiology and Society of Nuclear Medicine joint position statement: attenuation correction of myocardial perfusion SPECT scintigraphy. J Nucl Cardiol. 2004;11:229-230.

4. DePuey EG, Rozanski A. Using gated technetium-99m-sestamibi SPECT to characterize fixed myocardial defects as infarct or artifact. J Nucl Med. 1995;36: 952-955.

5. Garcia EV. SPECT attenuation correction: an essential tool to realize nuclear cardiology's manifest destiny. J Nucl Cardiol. 2007;14:16-24.

6. Thompson RC, Heller GV, Johnson LL, et al. Value of attenuation correction on ECG-gated SPECT myocardial perfusion imaging related to body mass index. J Nucl Cardiol. 2005;12:195-202.

7. Wolak A, Slomka PJ, Fish MB, Lorenzo S, Berman DS, Germano G. Quantitative diagnostic performance of myocardial perfusion SPECT with attenuation correction in women. J Nucl Med. 2008;49:915-922.

8. Germano G, Slomka PJ, Bermana DS. Attenuation correction in cardiac SPECT: the boy who cried wolf? J Nucl Cardiol. 2007;14:25-35.

9. Fricke H, Fricke E, Weise R, Kammeier A, Lindner O, Burchert W. A method to remove artifacts in attenuation-corrected myocardial perfusion SPECT introduced by misalignment between emission scan and CT-derived attenuation maps. J Nucl Med. 2004;45:1619-1625.

10. Nichols KJ. How serious a problem for myocardial perfusion assessment is moderate misregistration between SPECT and CT? J Nucl Cardiol. 2007;14: $150-152$.

11. Goetze S, Wahl RL. Prevalence of misregistration between SPECT and CT for attenuation-corrected myocardial perfusion SPECT. J Nucl Cardiol. 2007;14: 200-206.

12. Goetze S, Brown TL, Lavely WC, Zhang Z, Bengel FM. Attenuation correction in myocardial perfusion SPECT/CT: effects of misregistration and value of reregistration. J Nucl Med. 2007;48:1090-1095.

13. Matsunari I, Boning G, Ziegler SI, et al. Effects of misalignment between transmission and emission scans on attenuation-corrected cardiac SPECT. J Nucl Med. 1998;39:411-416.

14. Stone CD, McCormick JW, Gilland DR, Greer KL, Coleman RE, Jaszczak RJ. Effect of registration errors between transmission and emission scans on a SPECT system using sequential scanning. J Nucl Med. 1998;39:365-373.

15. Takahashi Y, Murase K, Higashino H, Mochizuki T, Motomura N. Attenuation correction of myocardial SPECT images with x-ray CT: effects of registration errors between X-ray CT and SPECT. Ann Nucl Med. 2002;16:431-435. 
16. Hudson HM, Larkin RS. Accelerated image reconstruction using ordered subsets of projection data. IEEE Trans Med Imaging. 1994;13:601-609.

17. Tsui B, Hu H, Gilland D, Gullberg GT. Implementation of simultaneous attenuation and detector response correction in SPECT. IEEE Trans Nucl Sci. 1988;35:778-783.

18. Hachamovitch R, Berman DS, Shaw LJ, et al. Incremental prognostic value of myocardial perfusion single photon emission computed tomography for the prediction of cardiac death: differential stratification for risk of cardiac death and myocardial infarction. Circulation. 1998;97:535543.

19. McQuaid SJ, Hutton BF. Sources of attenuation-correction artefacts in cardiac PET/CT and SPECT/CT. Eur J Nucl Med Mol Imaging. 2008;35: $1117-1123$.
20. Utsunomiya D, Nakaura T, Honda T, et al. Object-specific attenuation correction at SPECT/CT in thorax: optimization of respiratory protocol for image registration. Radiology. 2005;237:662-669.

21. Gould KL, Pan T, Loghin C, Johnson NP, Guha A, Sdringola S. Frequent diagnostic errors in cardiac PET/CT due to misregistration of CT attenuation and emission PET images: a definitive analysis of causes, consequences, and corrections. J Nucl Med. 2007;48:1112-1121.

22. Loghin C, Sdringola S, Gould KL. Common artifacts in PET myocardial perfusion images due to attenuation-emission misregistration: clinical significance, causes, and solutions. J Nucl Med. 2004;45:1029-1039.

23. Martinez-Möller A, Souvatzoglou M, Navab N, Schwaiger M, Nekolla SG. Artifacts from misaligned CT in cardiac perfusion PET/CT studies: frequency, effects, and potential solutions. J Nucl Med. 2007;48:188-193. 